\title{
Exergy Losses Relation with Driving Forces for Heat Transfer Process on Hot Plates Using Mathematical Programming
}

\author{
S. Ali Ashrafizadeh ${ }^{1}$, M. Amidpour ${ }^{2}$, N. Hedayat ${ }^{3}$ \\ ${ }^{1}$ Department of Chemical Engineering, College of Engineering, Dezful branch, \\ Islamic Azad University, Dezful, Iran. \\ ashrafi@iaud.ac.ir \\ ${ }^{2}$ Faculty of Mechanical Engineering, \\ K. N. Toosi University of Technology, Energy System Group. \\ amidpour@kntu.ac.ir \\ ${ }^{3}$ Department of Chemical Engineering, College of Engineering, Dezful branch, \\ Islamic Azad University, Dezful, Iran. \\ n.hedayat@yahoo.com
}

\begin{abstract}
The heat transfer on plates has many applications among which are the parallel plate heat exchangers. The application of second law of thermodynamics can lead to improvement in the quality of heat transfer process. The combination of design methods and second law analysis can provide an appropriate tool for the designer. The driving forces together with transfer coefficients, affect the heat transfer rate on one hand and given the relation of these forces with irreversibilities, they also influence the exergy losses on the other. Therefore, the driving forces can be used as a relation between heat transfer rate, transfer coefficients and exergy losses. The paper defines a new parameter, called here delivery impediment factor (DIF) which causes a difference in exergy change in the sink and source that lead to exergy losses. Exergy efficiency is expressed in relative driving force. A computer code has been developed to investigate various parameters that affect exergy losses within MATLAB environment. The method involved developing a sink and source model as well as basic relations in heat transfer on plate using mathematical programming. The effect of plate and fluid temperature on exergetic efficiency is shown in graphical form. Based on the results, a relation between the exergy efficiency and design parameters was obtained to make possible the thermodynamic analysis and design simultaneously.
\end{abstract}

Keywords: Hot Plates, Exergetic efficiency, Driving forces, Mathematical programming.

\section{Introduction}

For energy and environmental considerations, it would be crucial to apply a method by which to perform highly efficient heat transfer. The thermodynamic laws provide the designers and engineers with a powerful and efficient tool known as the exergy analysis. However, energy balance can provide useful information about the state of the system so far as the losses from the plant body and incomplete combustion are concerned. However, it doesn't display any difference between types of energy and doesn't consider the losses due to decrease of energy quality. By definition, exergy is referred to as the maximum shaft or electrical work in a reversible process when the system reaches the ambient conditions. Exergy analysis makes it possible to provide methods for thermodynamic development. However, economic and environmental considerations will determine the selection of final scenario.

There are massive literature materials describing the topics related to this subject. A. Bejan [1-2], for example, linked the principles of heat transfer to the second law of thermodynamics and entropy generation. Sargut et al. [3], T.J.Kotas [4], I. Dincer., A. Rosen [5], have taken gone further to develop and apply the concept of exergy analysis in various processes. H. Chang, S.C. Chuang [6], considered the intrinsic and extrinsic exergy losses in the processes. The first results from structural limitation whereas and the second from the transfer phenomena limitation. S. Mahmud, R. A. Fraser [7] performed the analysis of second law for convective heat transfer problem.

Heat exchangers are broadly used in a variety of industries, especially in the chemical industry. The main goal is to either increase cold fluid temperature or to decrease hot fluid temperature or (in some cases) doing both simultaneously. Temperature difference is the driving force in heat transfer causing irreversibility and entropy generation or exergy losses. 
Additionally, fluid friction is another reason for irreversibility in heat exchangers. Thus it is impossible to transfer the whole exergy from one fluid to another; as entropy generation and exergy losses are unavoidable in heat exchangers.

The second law analysis allows the heat exchanger designer to consider both the heat transfer rates between the fluids and the mechanical power expended to overcome fluid friction and to move the fluids through the heat exchanger simultaneously as the same is not possible with first law analysis. Therefore, there is a need for systematic design of heat exchangers using a second law based procedure. There have been many works on this subject in the literature. McClintock [8] carried out a pioneering work in this area by applying the irreversibility concept to heat exchanger design. Bejan [9] has explained two contributions to exergy loss i.e. one through heat transfer across finite temperature difference and the other through fluid friction in channels. Sarangi and Chowdhury [10] analyzed the entropy generation in a counter-flow heat exchanger and derived expressions in terms of relevant dimensionless parameters. Sekulic DP [11] investigated the entropy generation in a heat exchangers. Nag and Mukherjee [12] derived expression for entropy generation rate in connective heat transfer with constant wall temperature. Bejan [13] presented a broad spectrum of design optimization for simple co-current and countercurrent heat exchangers. Grazzini and Gori [14] carried out study based on entropy generation number for optimization heat exchanger design for counter heat exchangers. Ogulata et al. [15] analyzed behavior of parameters like optimum flow path length, dimensionless mass velocity, dimensionless heat transfer area for the crossflow plate type heat exchanger, operating with unmixed fluids. Sekulic [16] examined the influence of parameters like inlet temperature ratio, fluid flow heat capacity rate ratio, effectiveness on the quality of energy transformation for different type of heat exchangers. Mahmud and Fraser [17] performed the analysis of second law for heat exchangers with cylindrical annular space. Shah and Sekulic [18] gave a comprehensive explanation about the necessity of taking both first and second law into consideration for design of heat exchangers. Mahmud and Fraser [19] analyzed the forced convection in a circular duct for non-Newtonian fluids. Naphon [20] performed the analysis of second law for horizontal concentric tubes heat exchangers. Gupta and Das [21] analyzed the crossflow heat exchangers for non- uniform flow. Some studies have focused on plate heat exchangers. Das and Roetzel [22] presented exegetic analyzed of plate heat exchanger with axial dispersion in fluid that takes flow misdistribution and back mixing into consideration. Das and Roetzel [23] reported the natural of variation of different contributions to total exergy loss in heat exchanger with respect to axial dispersion parameters of the Peclet number and propagation velocity of dispersion wave. Mahmud and Fraser [24] analyzed the heat transfer and fluid flow inside channel with two parallel plates.

In every case of the heat transfer processes, there are two effective factors involved namely, the transfer coefficients and driving forces. The first factor is often related to material, geometry and dynamic properties of the system, whereas the driving forces are related to temperature distribution of system. In order to make a certain heat transfer rate, various ratios related to these two factors can be chosen. It has been established that the numerical value of these two factors will have certain effects on the amount of exergy losses. Most often accurate consideration of these two factors can increase the exegetic efficiency of system. Driving forces are essential to perform the real phenomena. These forces are created due to potential difference. Latter results in the irreversibility that in turn, lead to exergy losses. Knowledge of the relation between the exergy losses and driving forces can provide the designers and industrial users with appropriate informative picture of the method by which to minimize exergy losses or maximize economical benefits.

The present study attempts to establish a logical relation between the exegetic efficiency and these factors.

\section{Sink-Source Model Exergy Analysis in Heat Transfer Processes}

In all heat transfer processes which are performed above environment temperatures, the higher and lower temperature heat resource can be considered as a exergy source and sink respectively. According to the second law of thermodynamics, exergy sink can't absorb the whole source exergy. Thus, in the sink-source model, exergetic efficiency and exergy losses are defined as follows:

$$
\begin{gathered}
\eta=\frac{\Delta E X_{\sin k}}{\Delta E X_{\text {source }}} \\
E L=\Delta E X_{\text {source }}-\Delta E X_{\sin k}
\end{gathered}
$$


As can be seen from the above equations, if the exergy sink can absorb the higher exergy from source, the exergy losses and exergy efficiency will be lower and higher, respectively.

\section{General Exergy Analysis in Heat Transfer Processes}

Consider the two heat resource having different temperatures $\left(T_{1}>T_{2}\right)$ and the heat transfer rate between them equaling to "q". Based on these the flowing equations can be written to calculate the exergy variation:

$$
\begin{gathered}
\Delta E X_{\text {sowre }}=q\left(1-\frac{T_{0}}{T_{1}}\right) \\
\Delta E X_{\operatorname{sink}}=q\left(1-\frac{T_{0}}{T_{2}}\right)
\end{gathered}
$$

From “Eqs. (3\&4)." exergy losses can be calculated:

$$
E L=q T_{0}\left(\frac{T_{1}-T_{2}}{T_{1} T_{2}}\right)
$$

The rate of heat transfer can be calculated from "Eq. (6)."

$$
q=\frac{T_{1}-T_{2}}{R_{t h}}
$$

The closer the exergy variations in the source and sink are, the exergy losses are lower. The factor which influences the difference between the sink and source exergy variation is irreversibilities. This is known in the literature as the resistance against the exergy transfer. Since, in the heat transfer processes, the driving force is the temperature difference, which in turn has a direct relation with the irreversibilities. Thus it seems that these driving forces can be used to establish a logical relation between the sources and sink exergy variations. Based on the "Eq. (4)." it can be written:

$$
q=\frac{\Delta E X_{\sin k}}{1-T_{0} / T_{1}}
$$

By combining the "Eqs. (2\&6)." the following can be written:

$$
\Delta E X_{\sin k}=\Delta E X_{\text {sowre }}-q T_{0}\left(\frac{T_{1}-T_{2}}{T_{1} T_{2}}\right)
$$

By combining the "Eqs. (7\&8)." it can be written:

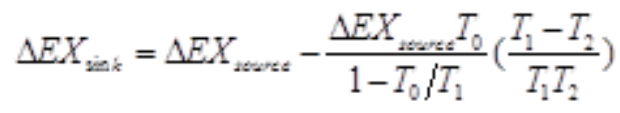

Arranging "Eq. (9)." it can be obtained:

$$
\Delta E X_{\sin k}=\frac{\Delta E X_{\text {sowre }}}{R_{E X}}
$$


The denominator in relation (10) is quantitative factors, which shows the reason for difference in the source and sink exergy change that is called here Delivery Impediment Factor (DIF).

$$
D I F=\frac{\left(T_{1}-T_{0}\right) / T_{1}}{\left(T_{2}-T_{0}\right) / T_{2}}
$$

The numerator and denominator in "Eq. (11)" are the relative distance of source and sink from the thermodynamics reference state, respectively. Clearly, the closer the right side of "Eq. (11)." is to one, the more exergy would be transferred from source to sink. This can be achieved if; the relative source distance from the thermodynamic reference is nearer to the relative sink distance from the same reference. As has been predicted, the 'DIF' is dependent on the driving force. The main point on the concept of 'DIF' is that it is influence by the value of the thermodynamic reference temperature $\left(\mathrm{T}_{0}\right)$. By the same token any variation in the reference temperature will have a corresponding change on 'DIF' value.

The other main point is the relationship between the 'DIF' and the exergetic efficiency of the heat transfer processes. By comparing the: "Eqs. (1\&11)." the following can be written:

$$
\eta=\frac{1}{D I F}=\frac{\left(T_{2}-T_{0}\right) / T_{2}}{\left(T_{1}-T_{0}\right) / T_{1}}
$$

The "Eq. (12)." is very useful as the exergetic efficiency can be obtained by knowing the source, sink and environment temperature. In order to achieve certain efficiency, an interactive relationship between the heat transfer and the driving forces should be established.

\section{Modeling of the Heat Transfer on Plates}

A model was developed in MATLAB environment. The objective function of which and its constraints are expressed in equation (16) as follows:

$$
\left\{\begin{array}{c}
\text { Objective function: } q=m_{f} \cdot C_{p_{f}} \cdot \Delta T_{f} \\
\text { Subject to: }\left\{\begin{array}{l}
A \leq A_{\max } \\
\eta_{E x}=\eta_{E x d}
\end{array}\right.
\end{array}\right.
$$

The aim of this model is to heat a certain fluid from lower to higher temperature $\left(T_{f 1} \& T_{f 2}\right)$. The constraints are the maximum permitted area and assumed exergetic efficiency.

The plate temperature could be determined by assuming a given exergetic efficiency and by using the environmental conditions and equation 11.

The exergy losses rate due to heat transfer can be estimated by the plate temperature and equation 5 .

Given the size of the area and assuming the flow type the required heat transfer coefficient can be estimated. Given the heat transfer coefficient, the fluid velocity over the plate can be estimated. The flow type can be determined by calculation of the Reynolds number. If the flow type does not correspond to the assumption, calculations for differing flows type should be repeated.

In this model the flow is assumed laminar, thus after calculating the heat transfer coefficient, using equation 6 and relating this coefficient with Nusselt number, the equations 14 to 17 are used to calculate the flow velocity.[25]

$$
\begin{gathered}
N u=0.664 \operatorname{Re}^{1 / 2} \operatorname{Pr}^{1 / 3} \\
N u=h . l / k \\
\operatorname{Re}=\rho . u \cdot d / \mu
\end{gathered}
$$

From equations 14 to 16 it can be written: 


$$
u=\left[\left(\frac{h l \mu^{\frac{1}{2}}}{k}\right) /\left(0.664 \rho^{1 / 2} l^{1 / 2} \operatorname{Pr}^{\frac{1}{3}}\right)\right]^{2}
$$

Having calculated the flow velocity, the Reynolds number is calculated. If the latter was greater than critical Reynolds number, the flow velocity can be estimated from Reynolds- Colburn similarity. The equations 18 to 20 show the relations used in this model [25].

$$
\begin{array}{cc}
\bar{C}_{f}=\frac{0.445}{\left(\log R e_{l}\right)^{2.584}}-\frac{A}{R e_{l}} & R e_{l}<10^{9} \\
\overline{S t} \cdot \operatorname{Pr}^{2 / 3}=\bar{C}_{f} / 2 & \\
\overline{S t} & =\frac{\bar{h}}{\rho \cdot u \cdot C_{P}}
\end{array}
$$

By combining the equation $18-20$ it can be written:

$$
u=\frac{2 h P r^{2 / 3}}{\rho C_{p}}\left(\frac{0.445}{\left(\log R e_{l}\right)^{2.584}}-\frac{A}{R e_{l}}\right)^{-1}
$$

The "A" parameter can be derived by table (1).[25]

Table 1: constant factor in Eq. 21.

\begin{tabular}{|l|l|}
\hline $\mathbf{R e}$ critical & A \\
\hline & \\
$3 \times 10^{5}$ & 1050 \\
$5 \times 10^{5}$ & 1742 \\
$10^{6}$ & 3340 \\
$3 \times 10^{6}$ & 8940 \\
\hline
\end{tabular}

By determining the fluid velocity the flow cross- section and thus the hydraulic diameter can be estimated. By using the latter in conjunction with the Darcy equation, the pressure loss estimated.

"Figure 1." shows the relation of exergetic efficiency to fluid temperature at the certain plate and reference temperature.

As can be observed from "Fig. 1." the more the relative distance plate and fluid temperature from the reference state are closer, the exergetic efficiency would be greater. Furthermore, any increase in the fluid temperature, will have a corresponding increase in the exergetic efficiency for any given plate temperature. On the other hand, the closer the plate and fluid temperatures are to each other, the exergy losses will be lower because the irreversiblities are lower. These will of course necessitate a decreased heat transfer rate.

What is crucial to consider in the exergetic efficiency study is the thermodynamic reference temperature. "Figure 2." shows the exergetic efficiency variations against the fluid temperature under different thermodynamic reference temperatures (in a fixed temperature for plate). 


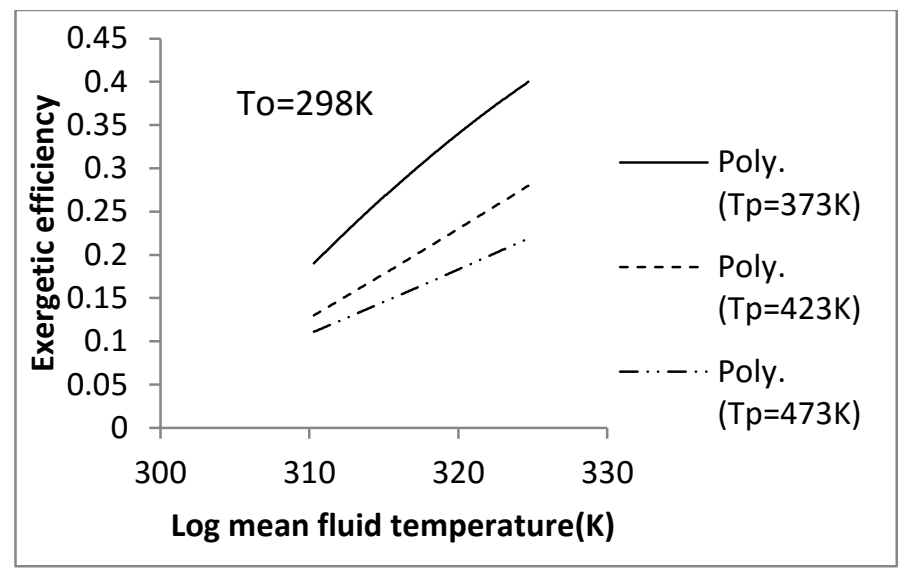

Fig. 1: Exergetic efficiency vs. fluid temperature at fixed reference temperature.

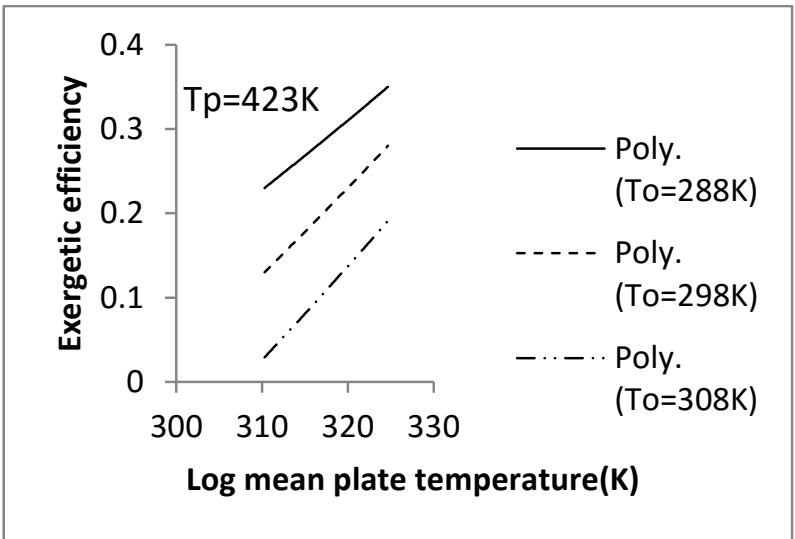

Fig. 2: Exergetic efficiency vs. fluid temperature at fixed plate temperature.

As was observed from the "Fig. 2." there is an inverse relation between the reference temperature and the exergy efficiency. In another words as the reference temperature decreases, exergy efficiency increases, and vice versa. "Figure 2." further suggests that any increase in the fluid temperature (implying a closed temperature between the plate and fluid), the effect of environment temperature is lower.

"Figure 3." shows the exergetic efficiency variations with regard to the plate temperature at different fluid temperature.

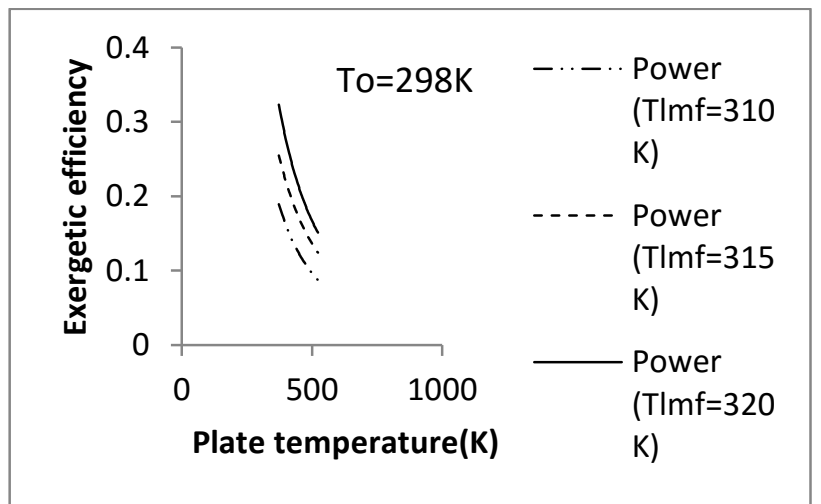

Fig. 3: Exergetic efficiency vs. plate temperature at fixed reference temperature.

Analysis of the "Fig. 3." indicates a direct relationship between the plate temperature and the distance between the plate and fluid temperature, but inverse relation with the exergetic efficiency. However, for a certain temperature of plate, the lesser the fluid temperature, the lesser would be the efficiency due to a greater distance. "Figure 4." shows the effect of 
the environmental temperature variations on the exergetic efficiency at the different plate temperature and certain temperature of fluid.

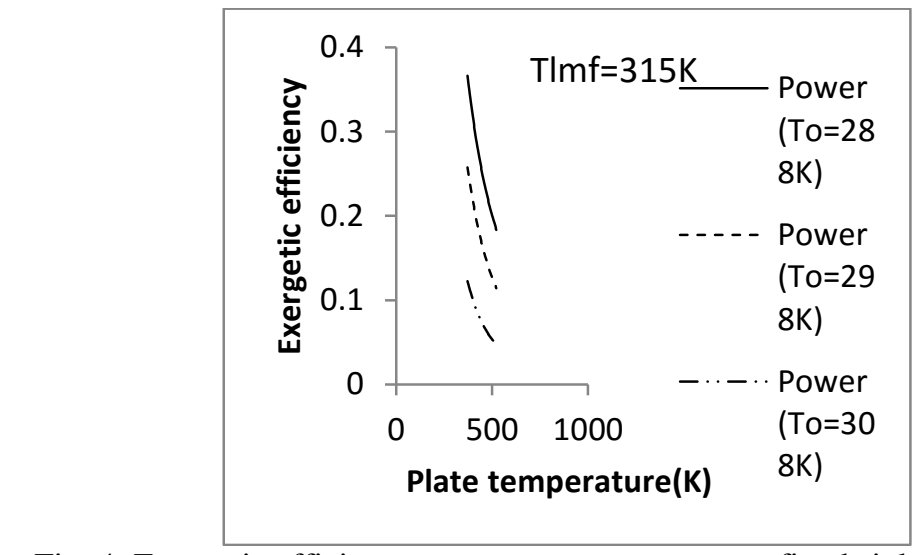

Fig. 4: Exergetic efficiency vs. source temperature at fixed sink temperature.

As can be observed from "Fig. 4." any increase in the reference temperature will have a corresponding decrease in the exergetic efficiency. What is crucial to consider is that the closer the temperatures between the fluid and plate, the effect of thermodynamic reference temperature would be negligible.

The question would be to determine the factors (apart from temperature) which are instrumental in achieving desirable exergetic efficiency. These can be mathematically derived at by using the equation 6 as follows:

$$
T_{2}=T_{1}-q \cdot R_{t h}
$$

Based on the "Eqs. (12\&13)" the exergetic efficiency functionality can be obtained:

$$
\eta=f\left(T_{1}, q \cdot R_{t h}\right)
$$

Equation (23) is a useful, as its application because it will make it possible to vary the heat resistances (which are related to the geometry and other dynamic system properties) in order to achieve higher exergetic efficiency at certain values of $\mathrm{T}_{1}$ and $\mathrm{q}$.

In the process, the "q" value can be calculated by the thermodynamic relation. The heat transfer is therefore a tool to fulfill the thermodynamics objectives. Depending on the type of problem, the source temperature is given in certain cases, whereas the sink temperature is given in others. Thus, the Eq. (14) can be varied and the efficiency functionality can be written as follows:

$$
\eta=f\left(T_{2}, q \cdot R_{t h}\right)
$$

Consider a system that it's source temperature is given. The aim is to obtain the relationship between exergetic efficiency and driving force for various value of "q.R $\mathrm{t}_{\mathrm{th}}$ ". These are illustrated by Fig. 5.

As is shown by Fig. 5 for a certain value of "q. $R_{t}$ " at the lower driving force value, the higher efficiency can be obtained. This incremental trend in driving forces leads to increase of irreversibilities and more exergy losses. "Equation (6)." further suggest that, the value of "q. $R_{t h}$ " is the same value of " $\Delta T$ ". It can be presumed that in a certain value of " $\Delta T$ ", the lesser the value of " $\Delta \mathrm{T} / \mathrm{T}_{1}$ ", the lower would be the efficiency increases. On the other hand, the higher the reservoir temperature, the higher would be the exergetic efficiency. 


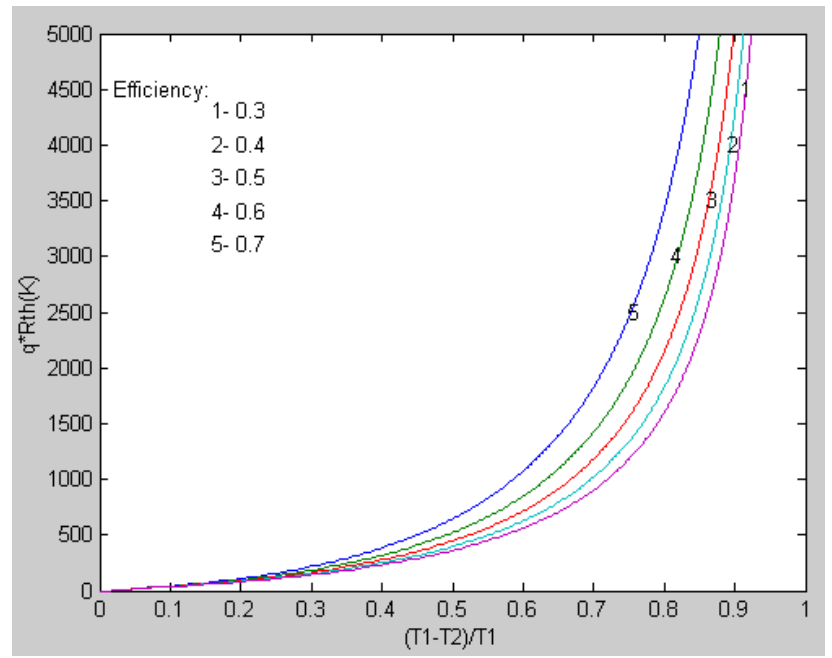

Fig. 5: Rate and resistance of heat transfer vs. relative driving force.

\section{Results}

The application of the sink-source model at exergy analysis of general heat transfer processes, made it possible for the present study to obtain the delivery impediment factor in exergy transfer from source to sink and exergy efficiency at heat transfer processes in terms of sink, source and reference temperature. The study also illustrated that the exergetic efficiency is the ratio of the relative distance of the sink from the thermodynamic reference to that of the source from the thermodynamic reference.

The delivery impediment factor parameter was found to be exactly the inverse of exergetic efficiency.

Comparisons of "Figs. $3 \& 1$ " suggest that under conditions of temperatures above the ambient, any rise in the fluid and fall in the plate temperatures will increase the exergy efficiency. On the other hand, as the relative plate and fluid distance are closer to the thermodynamic reference state; there would be a corresponding increase in the exergetic efficiency.

The comparison of "Figs.2\&4" showed that in the heat transfer processes above the ambient temperature, any increase in the reference temperature would lead to decreasing in the exergetic efficiency. Moreover, when the plate and fluid temperatures are very close to each other, the effect of thermodynamic reference is very negligible.

The relations $14 \& 15$ suggest that the exergetic efficiency can be considered as a function of the relative driving forces and the product of the heat transfer rate in the heat resistance, as is shown in "Fig 5." The above analyses made it possible to determine the temperatures, heat resistances and dynamic system in such a manner to attain appropriate exergetic efficiency.

\section{Reference}

[1] A. Bejan, Entropy Generation through Heat \& Fluid Flow. New York: Wiley, 1982.

[2] A. Bejan, G. Tsatsaronis, and M. Moran, Thermal Design \& Optimization. New York: Wiley, 1996.

[3] J. Szargut, D. R. Morris, and F. R. Steward, Exergy Analysis of Thermal, Chemical and Metallurgical Processes. New York, London: Hemisphere Publishing Corporation, 1988.

[4] T. J. Kotas, The Exergy Method of Thermal Plant Analysis. Malabar, Florida: Krieger Publishing Company, 1995.

[5] I. Dincer and M. A. Rosen, Exergy, Energy, Environment and Sustainable Development. Elsevier, 2007.

[6] H. Chang and S. C. Chuang, "Process analysis using the concept of intrinsic and extrinsic exergy losses," Energy, vol. 28, pp. 1203-1228, 2003.

[7] S. Mahmud and R. A. Fraser, "The second law analysis in fundamental convective heat transfer problems," International Journal of Thermal Sciences, vol. 42, pp. 177-186, 2003.

[8] F. A. McClintock, "The design of heat exchangers for minimum irreversibility," in Proceedings of ASME, 1951.

[9] A. Bejan, "General criterion for rating heat-exchanger performance," Int. J Heat Mass Transfer, vol. 21, pp. 655-8, 1978

[10] S. Sarangi, and K. Chowdhury, "On the generation of entropy in a counterflow heat exchangers," Cryogenics, vol. 22, pp. 63-5, 1982. 
[11] D. P. Sekulic, "Entropy generation in a heat exchanger," Heat Transfer Eng., vol. 7, no. 1-2, pp. 83-8, 1986.

[12] P. K. Nag and P. Mukherjee, "Thermodynamic optimization of convective heat transfer through a duct with constant wall temperature," Int. J. Heat Mass Transfer, vol. 2, pp. 401-5, 1987.

[13] A. Bejan, "The thermodynamic design of heat and mass transfer processes and devices," Int. J. Heat Fluid Flow, vol. 8, pp. 258-76, 1987.

[14] G. Grazzini and F. Gori, "Entropy parameters for heat exchanger design," Int. J. Heat Mass Transfer, vol. 31, pp. 254754, 1988.

[15] R. T. Ogulata, F. Doba, and T. Yilmaz, "Irreversibility analysis of cross flow heat exchangers," Energy Convers Manage, vol. 41, pp. 1585-99, 2000.

[16] D. P. Sekulic, "The second law quality of energy transformation in a heat exchanger," J. Heat Transfer, vol. 112, pp. 295-300, 1990.

[17] S. Mahmud and R. A. Fraser, "Second law analysis of heat transfer and fluid flow inside a cylindrical annular space," Exergy, an International Journal, vol. 2, pp. 322-329, 2002.

[18] R. K. Shah and D. P. Sekulic, Fundamentals of heat exchanger design. Hoboken, NJ: Wiley, p. 735-808, 2003.

[19] S. Mahmud and R. A. Fraser, "Second law analysis of forced convection in a circular duct for non-Newtonian ids," Energy, vol. 31, pp. 2226-2244, 2006.

[20] P. Naphon, "Second law analysis on the heat transfer of the horizontal concentric tube heat exchanger," International Communications in Heat and Mass Transfer, vol. 33, pp. 1029-1041, 2006.

[21] A. Gupta and S. K. Das, "Second law analysis of crossflow heat exchanger in the presence of axial dispersion in one fluid," Energy, vol. 32, pp. 664-72, 2007.

[22] S. K. Das and W. Roetzel, "Exergetic analysis of plate heat exchanger in precence of axial dispersion in fluid," Cryogenics, vol. 35, pp. 3-8, 1995.

[23] S. K. Das and W. Roetzel, "Second law analysis of a plate heat exchanger with an axial dispersive wave," Cryogenics, vol. 38, pp. 791-8, 1998.

[24] S. Mahmud and R. A. Fraser, "Thermodynamic analysis of flow and heat transfer inside channel with two parallel plates," Exergy, an International Journal, vol. 2, pp. 140-146, 2002.

[25] J. P. Holman, Heat transfer, 9th Ed. McGraw Hill, 2002. 